\title{
(p)ppGpp target ribosome assembly
}

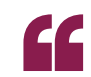

(p)ppGppinduced ... GTPase inactivation promotes bacterial survival on exposure to antimicrobials

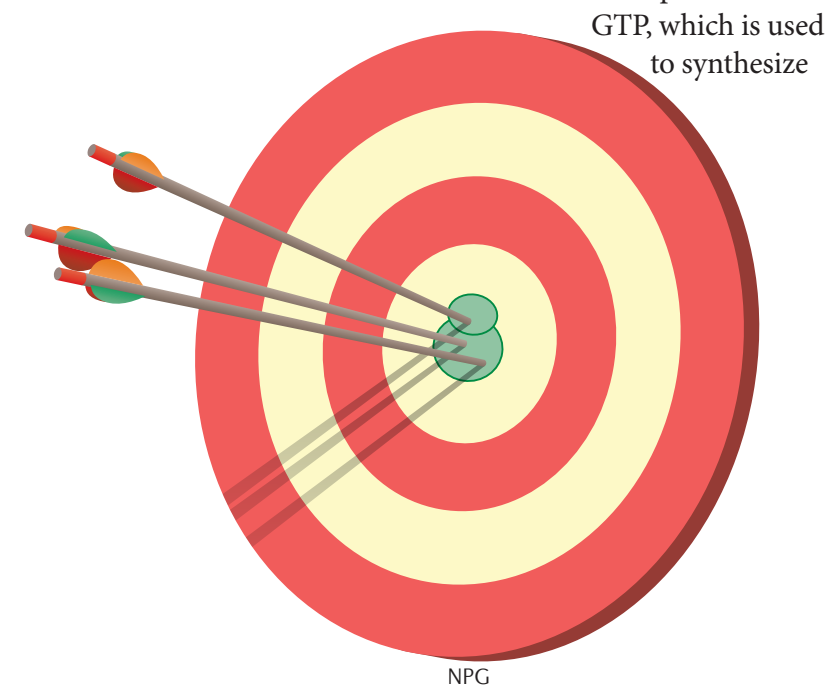

(p)ppGpp, was shown to decrease the transcription of mRNAs with a GTP-initiating nucleotide. Furthermore, (p)ppGpp binding inhibits HprT and Gmk, two enzymes that are involved in the GTP synthesis pathways. However, additional targets of (p)ppGpp are unknown.

To identify novel targets of (p)ppGpp, the authors used a screening assay that uses radiolabelled (p)ppGpp and tagged proteins from S. aureus. This strategy identified six (p)ppGpp-binding proteins: the known targets HprT and Gmk, and four novel proteins, RsgA, RbgA, Era and HflX. Notably, these novel proteins are homologous to GTPases that are involved in ribosomal biogenesis in other bacteria, which prompted the authors to investigate whether they had GTPase activity and participated in ribosome assembly. Indeed, recombinant versions of the four proteins were able to hydrolyse GTP, and this enzymatic activity was inhibited in the presence of (p)ppGpp and increased in the presence of 705 ribosomes.

To investigate the role of these proteins in vivo, the authors focused on RsgA and generated two mutant strains, one lacking $r s g A$ and another with a mutation in the GTPase domain of RsgA that abolished its enzymatic activity. Importantly, both mutants had substantial growth defects, and comparing the ribosome profiles of the mutants with wild-type $S$. aureus revealed that the mutants had decreased levels of mature $70 \mathrm{~S}$ ribosomes and accumulated the $30 \mathrm{~S}$ and $50 \mathrm{~S}$ subunit components.
Collectively, these data suggest that the GTPase activity of RsgA is necessary for the assembly of the $30 \mathrm{~S}$ and 50S subunits into mature $70 \mathrm{~S}$ ribosomes, and that this activity is inhibited by (p)ppGpp, which promotes slow growth.

As (p)ppGpp-induced slow growth has been linked to tolerance to antimicrobials in Gram-negative bacteria, the authors then investigated whether the same was true in S. aureus. Indeed, both mutants showed increased survival compared with wild-type bacteria when grown in the presence of penicillins, vancomycin or ciprofloxacin, which demonstrates that GTPase inactivation promotes bacterial survival on exposure to antimicrobials.

Together, these data establish a novel mechanism by which (p)ppGpp regulates the stringent response in $S$. aureus by binding to and inhibiting several GTPases, thereby interfering with ribosome assembly, slowing growth and promoting tolerance to antimicrobials. Importantly, the authors also showed that homologues of these GTPases that are present in Bacillus subtilis and Enterococcus faecalis can bind to (p)ppGpp, which suggests that this mechanism may be widely distributed among Gram-positive bacteria.

Cláudio Nunes-Alves

ORIGINAL ARTICLE Corrigan, R. M. et al. ppGpp negatively impacts ribosome assembly affecting growth and antimicrobial tolerance in Grampositive bacteria. Proc. Natl. Acad Sci. USA http:// dx.doi.org/10.1073/pnas.1522179113 (2016) FURTHER READING Hauryliuk, V. et al. Recent functional insights into the role of (p)ppGpp in bacterial physiology. Nat. Rev. Microbiol. 13, 298-309 (2015) 